Journal of Acute Disease

journal homepage: www.jadweb.org

\title{
Persistent fever during treatment of a pregnant woman with acute pyelonephritis
}

\author{
Hasan Tahsin Gozdas ${ }^{1 *}$, Mehmet Dinek ${ }^{2}$
}

${ }^{\prime}$ Department of Infectious Diseases and Clinical Microbiology, Dr. Munif Islamoglu Kastamonu State Hospital, Kastamonu, Turkey

${ }^{2}$ Department of Urology, Dr. Munif Islamoglu Kastamonu State Hospital, Kastamonu, Turkey

\begin{tabular}{l} 
ARTICLE INFO \\
\hline Article history: \\
Received 9 Oct 2016 \\
Received in revised form 27 Oct 2016 \\
Accepted 8 Nov 2016 \\
Available online 8 Dec 2016 \\
\hline
\end{tabular}

Keywords:

Persistent fever

Acute pyelonephritis

Pregnancy

Double-J ureteral stent

\section{ABSTRACT}

Acute pyelonephritis is a serious infection in pregnancy. It is presented with fever, shaking chills and flank pain. Intravenous hydration and antimicrobial therapy are sufficient in the treatment unless pyelonephritis is complicated. In case of fever persisting for more than $48 \mathrm{~h}$ despite appropriate antimicrobial treatment, a possible complication such as urinary tract obstruction, abscess or phlegmon should be considered. Here, we present an 18 -year-old pregnant woman with acute pyelonephritis whose persistent fever returned to normal after double-J ureteral stent was placed even if she had no finding of such a complication.

\section{Introduction}

Acute pyelonephritis is a serious complication of pregnancy. It is more frequently seen in the second trimester and usually affets the right kidney. Nulliparity and young age are important risk factors. Fever, shaking chills and flank pain are the main symptoms. Escherichia coli is the most causative agent, whereas Klebsiella pneumoniae, Enterobacter and Proteus spp. and Gram positive organisms can be isolated as rare responsible agents. Intravenous hydration and antimicrobial therapy are the mainstay of treatment[1,2]. Ultrasound is helpful in detecting complications such as urinary tract obstruction (e.g. calculi), abscess or phlegmon. If an obstruction complicates acute pyelonephritis treatment, then double-J ureteral stent insertion is considered[3-5].

\section{Case report}

An 18-year-old (26-week-pregnant, nullipara) woman applied to emergency department because of fever, shaking chills and right flank pain for about a week. Her past medical history and family history were normal. Physical examination revealed fever: $39.4{ }^{\circ} \mathrm{C}$;

*Corresponding author: Hasan Tahsin Gozdas, Department of Infectious Diseases and Clinical Microbiology, Dr. Munif Islamoglu Kastamonu State Hospital, Kastamonu, Turkey.

Tel: +903662141053

E-mail: dr.htgozdas@yahoo.com.tr

The journal implements double-blind peer review practiced by specially invited international editorial board members. heart rate: $88 / \mathrm{min}$; blood pressure: $120 / 80 \mathrm{mmHg}$ and respiratory rate: $16 / \mathrm{min}$. The chest was clear on auscultation and cardiovascular examination revealed normal heart sounds. Costovertebral tenderness was positive on the right side. Gynecologic examination was normal. Laboratory findings were white blood count (WBC): $22.33 \mathrm{~K} / \mu \mathrm{L}, \mathrm{C}$-reactive protein (CRP): $63.9 \mathrm{mg} / \mathrm{L}$, urinanalysis: 214 leukocytes/high power field (HPF), renal functions and liver enzymes were normal. Abdominal ultrasound showed right grade 2 hydronephrosis (anterior-posterior diameter of renal pelvis: $20 \mathrm{~mm}$ ), and left grade 1 hydronephrosis (anterior-posterior diameter of renal pelvis: $12 \mathrm{~mm}$ ). She was diagnosed with acute pyelonephritis and admitted to inpatient clinic. Intravenous fluid resusitation was initiated. After blood and urine cultures were obtained, ceftriaxone $1 \times 2 \mathrm{~g}$ intravenously was started empirically. At $48 \mathrm{~h}$ of ceftriaxone treatment, her clinical status and laboratory findings exacerbated (WBC: $24.16 \mathrm{~K} / \mu \mathrm{L}$, CRP: $214.8 \mathrm{mg} / \mathrm{L}$, urinanalysis: 277 leukocytes/HPF, 4 erythrocye/HPF). Then, ceftriaxone was switched to meropenem $3 \times 1 \mathrm{~g}$ intravenously. Extended spectrum $\beta$ lactamase producing Klebsiella pneumoniae grew in urine culture. Blood culture showed no growth. Due to sensitivity on antibiogram, meropenem was continued. At $48 \mathrm{~h}$ of meropenem treatment, blood tests showed significant improvement (WBC: $7.38 \mathrm{~K} / \mu \mathrm{L}, \mathrm{CRP}: 174.5 \mathrm{mg} / \mathrm{L}$ ), urinanalysis was normal, and urine culture was sterile. However, she did not show adequate clinical improvement and her fever persisted. Despite culture negativity and laboratory improvement, she was still febrile at the 5th day of meropenem treatment. Abdominal ultrasound was repeated for 
a possible complication. However, no complication was found. In contrast, a mild decrease in right hydronephrosis was detected (anterior-posterior diameter of renal pelvis: $16 \mathrm{~mm}$ ). Although no finding in favor of obstruction was found, double-J ureteral stent was placed to the right ureter to improve urine flow. Interestingly, she became afebrile from the next day. Then, meropenem was continued for 10 days. She recovered completely and discharged with a recommendation of outpatient control.

\section{Discussion}

Pregnancy predisposes to acute pyelonephritis which can further be complicated by obstruction (e.g. calculi), abscess or phlegmon. Intravenous hydration and antimicrobial therapy are sufficient in the treatment unless pyelonephritis is complicated. Complications such as urinary tract obstruction (e.g. calculi), abscess or phlegmon can be identified by ultrasound[1-4].

During normal pregnancy, peristalsism of the ureters decrease and most women in third trimester exhibit significant ureteral dilatation. This hydroureter is related to muscle-relaxing effect of increased progesterone and mechanical obstruction of the ureters by the uterus. At the same time, the enlarged uterus compresses the urinary bladder, thereby increasing the intravesical pressure which can result in vesico-ureteral reflux and retention of urine in the bladder. Urinary stasis and impairment of physiological anti-reflux mechanism inversely affect normal urine flow[6].

Although renal pelvis dilation is normally seen in pregnancy, severe dilation can be a sign of obstruction as well. Twickler et al.[7] investigated renal pelvis dilatation in antepartum pyelonephritis by ultrasonography. They compared renal pelvis dimensions of 24 pregnant women with pyelonephritis and healthy control subjects matched for gestational age, parity and race. They found that renal pelvicalyceal system dilation was significantly increased in antepartum pyelonephritis as compared with normal physiologic dilation of pregnancy $(17 \mathrm{~mm}$ v.s. $8 \mathrm{~mm}, P<0.001)$. Dilation of renal pelvis might prepare a suitable medium for the development of acute pyelonephritis in pregnancy.

Seidman et al.[8] retrospectively reviewed 171 consecutive pregnant women with pyelonephritis. Renal pelvis dilation was mild $(6-10 \mathrm{~mm})$ in $25(33.3 \%)$, moderate $(11-15 \mathrm{~mm})$ in $16(21.3 \%)$ and severe $(16 \mathrm{~mm})$ in $8(10.7 \%)$ of their patients. Duplicated collecting systems and renal calculi were each found in 2 (2.7\%) of them. All of them were treated conservatively, none of them required ureteral stent. In our patient, anterior-posterior diameter of right renal pelvis was $20 \mathrm{~mm}$ at admission, however it decreased to $16 \mathrm{~mm}$ at the 5th day of hospitalisation. Although a mild decrease in renal pelvis dilation was achieved with conservative treatment in our patient, her fever persisted during five days despite appropriate antimicrobial therapy.

If fever continues or clinical improvement is not achieved by 48 to $72 \mathrm{~h}$ of treatment, urinary tract obstruction (e.g. lithiasis, congenital or acquired structural changes within the urinary tract) or another complication (e.g. intraabdominal infection, perirenal abscess, phlegmon) must be considered[9,10]. Our patient's fever persisted at the 5th day of appropriate antimicrobial treatment. We repeated ultrasonography for a possible complication. However, we could not identify any complicating factor such as calculi, abscess or phlegmon.

Cystoscopic placement of double-J ureteral stent is recommended when there is obstruction in the urinary tract[3,5]. Although no finding in favor of obstruction was found in our patient, double-J ureteral stent was placed because of persistent fever. She became afebrile from the next day.

In our patient, we obtained culture negativity and laboratory improvement, but we could not obtain fever response during five days. We could not identify any complicating factor that could cause persistence of fever. Our patient might have benefited from double-J stent application probably due to improvement of mechanical obstruction caused by the pregnancy itself. Thus, we concluded that double-J ureteral stent insertion may be useful in acute pyelonephritis treatment during pregnancy even if a clear obstruction could not be found.

\section{Conflict of interest statement}

The authors report no conflict of interest.

\section{Acknowledgment}

We would like to thank Dr. Mensure Tonguc for her valuable contributions in the management of this patient.

\section{References}

[1] Matuszkiewicz-Rowińska J, Małyszko J, Wieliczko M. Urinary tract infections in pregnancy: old and new unresolved diagnostic and therapeutic problems. Arch Med Sci 2015; 11: 67-77.

[2] Hill JB, Sheffield JS, McIntire DD, Wendel GD Jr. Acute pyelonephritis in pregnancy. Obstet Gynecol 2005; 105: 18-23.

[3] Butler EL, Cox SM, Eberts E, Cunningham FG. Symptomatic nephrolithiasis complicating pregnancy. Obstet Gynecol 2000; 96: 753-6.

[4] Rafi J, Smith RB. Acute lobar nephronia in pregnancy: a rarely reported entity in obstetric renal medicine. Arch Gynecol Obstet 2012; 286: 797-8.

[5] Semins MJ, Matlaga BR. Management of stone disease in pregnancy. Cur Opin Urol 2010; 20: 174-7.

[6] Jeyabalan A, Lain KY. Anatomic and functional changes of the upper urinary tract during pregnancy. Urol Clin North Am 2007; 34: 1-6.

[7] Twickler D, Little BB, Satin AJ, Brown CE. Renal pelvicalyceal dilation in antepartum pyelonephritis: ultrasonographic findings. Am J Obstet Gynecol 1991; 165: 1115-9.

[8] Seidman DS, Soriano D, Dulitzki M, Heyman Z, Mashiach S, Barkai G. Role of renal ultrasonography in the management of pyelonephritis in pregnant women. J Perinatol 1998; 18: 98-101.

[9] Standard treatment of pyelonephritis in pregnancy involves parenteral antibacterials and intravenous hydration. Drugs Ther Perspect 2011; 27: 13-7.

[10] Nicolle LE, Bradley S, Colgan R, Rice JC, Schaeffer A, Hooton TM, et al. Infectious Diseases Society of America guidelines for the diagnosis and treatment of asymptomatic bacteriuria in adults. Clin Infect Dis 2005; 40: 643-54. 